\title{
Thumbprinting Locally Advanced Pancreatic Cancer: Have We Developed the Optimal Staging System?
}

\author{
Caitlin A. Hester, MD, and Matthew H. G. Katz, MD \\ Department of Surgical Oncology, The University of Texas MD Anderson Cancer Center, Houston, TX
}

Locally advanced pancreas cancers (LAPC) are broadly defined by significant interfaces between the primary pancreatic tumor and major abdominal blood vessels in the absence of detectable metastases. However, like all pancreatic adenocarcinomas, the majority of LAPC is associated with occult extrapancreatic cancer, which limits the role of local therapies. LAPC has historically been considered inoperable because pancreatectomy has been largely ineffective at prolonging longevity and has been associated with prohibitive rates of perioperative morbidity and mortality. ${ }^{1,2}$ Increasingly, however, multidisciplinary teams have recognized a potential role for pancreatectomy in patients with LAPC who have a robust physiologic profile and radiographically localized disease following induction chemotherapy and/or (chemo)radiation. ${ }^{3} \mathrm{~A}$ nuanced staging system that acknowledges the clinical heterogeneity within LAPC has yet to be widely adopted.

In this issue of Annals of Surgical Oncology, Fromer and colleagues propose a novel staging classification for LAPC designed to facilitate communication, standardize enrollment of patients into clinical trials, and select patients for surgery. ${ }^{4}$ The authors build upon existing anatomic criteria for LAPC to create seven subclasses based on the radiographic relationships between primary pancreatic tumors and the major abdominal vasculature. The authors support their classification through a systematic review of available perioperative outcome data for each subclass and supplement these data with expert opinion. The authors

(C) Society of Surgical Oncology 2021

First Received: 23 April 2021

Accepted: 7 May 2021;

Published Online: 21 May 2021

M. H. G. Katz, MD

e-mail: mhgkatz@mdanderson.org emphasize differences in technical complexity of and perioperative risk associated with resection of tumors within each subclass.

We fully agree with Fromer and colleagues that a more granular staging classification is necessary to facilitate multidisciplinary communication and ensure enrollment of homogeneous patient populations into clinical trials. ${ }^{5}$ Their proposal highlights the anatomic variability of LAPC and distinguishes tumors adjacent to a single surgically relevant blood vessel from those adjacent to multiple vessels. However, their taxonomy is limited by imprecision in anatomic terminology and an assumption that all LAPC tumors would necessitate vascular resection at pancreatectomy. For anatomic staging, we strongly discourage the use of radiographic terms such as vascular "involvement," "abutment," or "encasement," which introduce ambiguity and are subject to bias. Furthermore, these terms convey very little about true histopathologic vessel wall invasion or the need for vascular resection at pancreatectomyparticularly following induction chemotherapy and/or (chemo)radiation therapy. Indeed, we and others have shown that tumors that are suggested by cross-sectional imaging studies to "involve" veins or arteries often do not and have described techniques that may be used to resect tumors without subjecting patients to the potential morbidity of vascular resection. ${ }^{6,7}$ Ultimately, this imprecision could lead to heterogeneity in enrollment of patients to clinical treatment trials and artifact in terms of these treatments' perceived effects. ${ }^{8}$

Furthermore, any clinical staging system that is designed to clarify the role of surgery for patients with LAPC and is based on tumor anatomy alone is by design limited in impact. In the NEOLAP phase II trial, the resection rate following induction chemotherapy for LAPC_-defined using imaging criteria, surgical exploration, or both-was $32 \% .^{3}$ Obvious staging bias 
notwithstanding, this resection rate is among the highest for patients with LAPC ever reported in the literature. ${ }^{3}$ Resection rates reported in other major trials include $<5 \%$ in the LAP07 trial and SCALOP trial and $15 \%$ in the LAPACT trial. ${ }^{9-11}$ And, while patients who undergo R0 resection may enjoy longevity, patients in whom margins are positive may not. In the NEOLAP trial, for example, the median overall survival of patients who underwent R0 pancreatectomy was 40 months, but the median overall survival of patients who underwent R1 pancreatectomy, which occurred in $>30 \%$ of operations, was similar to that of patients who did not undergo pancreatectomy at all. ${ }^{3}$ Although surgery can and should be considered in highly selected patients following induction chemotherapy and/or (chemo)radiation therapy, these data emphasize that surgical resection should not be considered the goal of therapy for patients with LAPC (or, we would argue, for patients with any stage of pancreatic cancer). The goals, instead, should be longevity and quality of life. Focusing on surgical resection as a therapeutic objective risks subjecting patients to highly morbid technical exercises that, for most, are unlikely to change the natural history of their disease.

To predict longevity and guide the treatment course, it is our practice to rely less on the initial anatomic clinical stage and more on longitudinal data collected over time to better capture the "thumbprint" of a cancer, the so-called cancer biology. Clinical stage should drive initial treatment, but subsequent therapies should be driven by data, such as radiographic, radiomic, and serologic markers of response, as they accumulate over the course of treatment. ${ }^{12}$ Ultimately, we consider pancreatectomy only in those patients who appear to have locally dominant, treatment-responsive cancer; who are physiologically robust; who understand the risk associated with these extensive operations; and who are believed, on the basis of a multidisciplinary discussion, to have the potential for R0 resection. We strongly believe that staging should take into account more than can be defined by a single anatomic time stamp and must capture a panoramic view of the natural history of the disease in each individual patient.

Certainly, systemic treatments have evolved for the better, and more patients with LAPC should be considered for pancreatectomy than were considered in the past. This heterogeneous stage calls for more granular staging definitions to facilitate multidisciplinary communication and trial enrollment. However, chemotherapy remains the mainstay of therapy for all patients with LAPC, and surgery should be used only for those with a strong likelihood of benefit-a likelihood that cannot be predicted on the basis of tumor anatomy alone. Therefore, any staging model that aims to predict longevity or operative potential must include patient and biologic factors in its design.
ACKNOWLEDGEMENTS We thank Stephanie Deming, Research Medical Library, MD Anderson Cancer Center, for editing this editorial.

\section{REFERENCES}

1. Balaban EP, Mangu PB, Yee NS. Locally advanced unresectable pancreatic cancer: American Society of Clinical Oncology clinical practice guideline summary. J Oncol Pract. 2017;13(4):265-9.

2. Gulhati P, Prakash L, Katz MHG, Wang X, Javle M, Shroff R, Fogelman D, Lee JE, Tzeng CD, Lee JH, et al. First-line gemcitabine and nab-paclitaxel chemotherapy for localized pancreatic ductal adenocarcinoma. Ann Surg Oncol. 2019;26(2):619-27.

3. Kunzmann V, Siveke JT, Algul H, Goekkurt E, Siegler G, Martens U, Waldschmidt D, Pelzer U, Fuchs M, Kullmann F, et al. Nab-paclitaxel plus gemcitabine versus nab-paclitaxel plus gemcitabine followed by FOLFIRINOX induction chemotherapy in locally advanced pancreatic cancer (NEOLAP-AIO-PAK0113): a multicentre, randomised, phase 2 trial. Lancet Gastroenterol Hepatol. 2021;6(2):128-38.

4. Fromer M, Hawthorne J, Philips P, Egger M, Scoggins C, McMasters K, Martin R. An improved staging system for locallyadvanced pancreatic cancer: a critical need in the multidisciplinary era. Ann Surg Oncol. 2021. https://doi.org/10.1245/ s10434-021-10174-z.

5. Katz MH, Marsh R, Herman JM, Shi Q, Collison E, Venook AP, Kindler HL, Alberts SR, Philip P, Lowy AM, et al. Borderline resectable pancreatic cancer: need for standardization and methods for optimal clinical trial design. Ann Surg Oncol. 2013;20(8):2787-95.

6. Tran Cao HS, Balachandran A, Wang H, Nogueras-Gonzalez GM, Bailey CE, Lee JE, Pisters PW, Evans DB, Varadhachary G, Crane $\mathrm{CH}$, et al. Radiographic tumor-vein interface as a predictor of intraoperative, pathologic, and oncologic outcomes in resectable and borderline resectable pancreatic cancer. $J$ Gastrointest Surg. 2014;18(2):269-78 (discussion 278).

7. Habib JR, Kinny-Koster B, van Oosten F, Javed AA, Cameron JL, Lafaro KJ, Burkhart RA, Burns WR, He J, Thompson ED, et al. Periadventitial dissection of the superior mesenteric artery for locally advanced pancreatic cancer: Surgical planning with the "Halo sign" and "String sign". Surgery. 2020;169(5): 1026-1031.

8. Katz MH, Shi Q, Ahmad SA, Herman JM, Marsh RDW, Collisson E, Schwartz L, Frankel W, Martin R, Conway W, et al. Preoperative modified FOLFIRINOX treatment followed by capecitabine-based chemoradiation for borderline resectable pancreatic cancer: alliance for clinical trials in oncology trial a021101. JAMA Surg. 2016;151(8):e161137.

9. Hammel P, Huguet F, van Laethem JL, Goldstein D, Glimelius B, Artru P, Borbath I, Bouche O, Shannon J, Andre T, et al. Effect of chemoradiotherapy vs chemotherapy on survival in patients with locally advanced pancreatic cancer controlled after 4 months of gemcitabine with or without erlotinib: the LAP07 randomized clinical trial. JAMA. 2016;315(17):1844-53.

10. Mukherjee S, Hurt CN, Bridgewater J, Falk S, Cummins S, Wasan H, Crosby T, Jephcott C, Roy R, Radhakrishna G, et al. Gemcitabine-based or capecitabine-based chemoradiotherapy for locally advanced pancreatic cancer (SCALOP): a multicentre, randomised, phase 2 trial. Lancet Oncol. 2013;14(4):317-26.

11. Philip PA, Lacy J, Portales F, Sobrero A, Pazo-Cid R, Manzano Mozo JL, Kim EJ, Dowden S, Zakari A, Borg C, et al. Nabpaclitaxel plus gemcitabine in patients with locally advanced pancreatic cancer (LAPACT): multicentre, open-label phase 2 study. Lancet Gastroenterol Hepatol. 2020;5(3):285-94. 
12. Perri G, Prakash L, Wang H, Bhosale P, Varadhachary GR, Wolff R, Fogelman D, Overman M, Pant S, Javle M, et al. Radiographic and serologic predictors of pathologic major response to preoperative therapy for pancreatic cancer. Ann Surg. 2021;273(4):806-13
Publisher's Note Springer Nature remains neutral with regard to jurisdictional claims in published maps and institutional affiliations. 